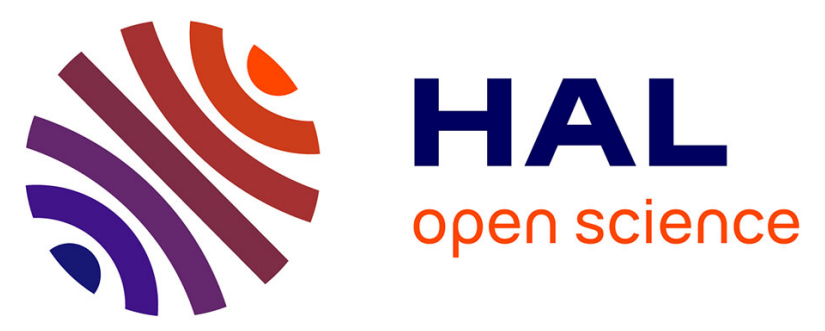

\title{
Impact of the Size of Modules on Target Acquisition and Pursuit for Future Modular Shape-changing Physical User Interfaces
}

Laura Pruszko, Yann Laurillau, Benoît Piranda, Julien Bourgeois, Céline

Coutrix

\section{To cite this version:}

Laura Pruszko, Yann Laurillau, Benoît Piranda, Julien Bourgeois, Céline Coutrix. Impact of the Size of Modules on Target Acquisition and Pursuit for Future Modular Shape-changing Physical User Interfaces. Proceedings of the 2021 International Conference on Multimodal Interaction (ICMI '21), Oct 2021, Montréal, Canada. 10.1145/3462244.3479936 . hal-03325220

\section{HAL Id: hal-03325220 \\ https://hal.science/hal-03325220}

Submitted on 30 Aug 2021

HAL is a multi-disciplinary open access archive for the deposit and dissemination of scientific research documents, whether they are published or not. The documents may come from teaching and research institutions in France or abroad, or from public or private research centers.
L'archive ouverte pluridisciplinaire HAL, est destinée au dépôt et à la diffusion de documents scientifiques de niveau recherche, publiés ou non, émanant des établissements d'enseignement et de recherche français ou étrangers, des laboratoires publics ou privés. 


\section{Impact of the Size of Modules on Target Acquisition and Pursuit for Future Modular Shape-changing Physical User Interfaces}

\author{
Laura Pruszko \\ Yann Laurillau \\ first.last@univ-grenoble-alpes.fr \\ Université Grenoble Alpes
}

\author{
Benoît Piranda \\ Julien Bourgeois \\ first.last@femto-st.fr \\ Univ. Bourgogne Franche-Comté, \\ Institut FEMTO-ST, CNRS
}

\author{
Céline Coutrix \\ celine.coutrix@imag.fr \\ CNRS, Université Grenoble Alpes
}

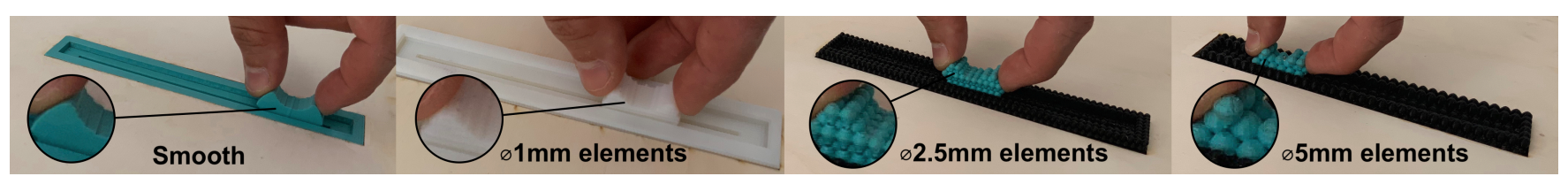

Figure 1: The four sliders used to investigate the impact of the size of future modules constituting the PUI: (from left to right) a current smooth slider and three sliders made of mock-up modules of $\varnothing 1 \mathrm{~mm}, \varnothing 2.5 \mathrm{~mm}$ and $\varnothing 5 \mathrm{~mm}$.

\begin{abstract}
Shape-changing User Interfaces (UIs) explore the ability of a UI to change its physical shape to support multiple interaction modalities for users' input and/or system's output. An approach currently studied to implement such interfaces at a high resolution is based on mm-sized, round, and self-actuated modules. The problem we tackle in this paper is to find the range of usable sizes of such modules, to better inform the trade-off between usability and technological feasibility. We assessed four sliders in a controlled user study: a standard slider and three sliders made of mock-up rounded modules of $\varnothing 1 \mathrm{~mm}, \varnothing 2.5 \mathrm{~mm}$, and $\varnothing 5 \mathrm{~mm}$. Experimental results show that (1) $\varnothing 5 \mathrm{~mm}$ modules significantly impair performance for the pursuit task and subjective perception for both tasks, (2) performance increases when the size of modules decreases, but (3) users reportedly enjoyed the haptic feedback provided by $\varnothing 1 \mathrm{~mm}$ to $\varnothing 2.5 \mathrm{~mm}$ modules. These results provide deeper understanding on the impact of the size of modules on performance and subjective perception to inform current technological development of physical user interfaces made of small robotic modules.
\end{abstract}

\section{CCS CONCEPTS}

- Human-centered computing $\rightarrow$ Empirical studies in HCI.

\section{KEYWORDS}

Physical interaction, Slider, Target acquisition, Target pursuit, Modular Robotics, Shape-changing Interfaces, Modularity

Permission to make digital or hard copies of all or part of this work for personal or classroom use is granted without fee provided that copies are not made or distributed for profit or commercial advantage and that copies bear this notice and the full citation on the first page. Copyrights for components of this work owned by others than ACM must be honored. Abstracting with credit is permitted. To copy otherwise, or republish, to post on servers or to redistribute to lists, requires prior specific permission and/or a fee. Request permissions from permissions@acm.org.

ICMI '21, October 18-22, 2021, Montréal, QC, Canada

(C) 2021 Association for Computing Machinery.

ACM ISBN 978-1-4503-8481-0/21/10 . \$ \$15.00

https://doi.org/10.1145/3462244.3479936

\section{ACM Reference Format:}

Laura Pruszko, Yann Laurillau, Benoît Piranda, Julien Bourgeois, and Céline Coutrix. 2021. Impact of the Size of Modules on Target Acquisition and Pursuit for Future Modular Shape-changing Physical User Interfaces. In Proceedings of the 2021 International Conference on Multimodal Interaction (ICMI '21), October 18-22, 2021, Montréal, QC, Canada. ACM, New York, NY, USA, 11 pages. https://doi.org/10.1145/3462244.3479936

\section{INTRODUCTION}

Recent work on shape-changing User Interfaces (UIs) explored the ability of a UI to change its physical shape to support multiple interaction modalities [11] for users' input and system's output [2]. Shape-changing UIs offer the benefits of physicality and software dynamics, and enable, e.g., the unique support of adaptative affordance [2]. Examples include a slider of variable length to compromise between performance and footprint [19] (Figure 2a).

One possible approach to implement shape-changing UIs is based on modules. Modules are small building block elements, which can rearrange or be rearranged to form the UI. Such modules can be robotic, i.e., microelectromechanical modules embedding computational capabilities (e.g., Catoms [94], MBlocks [80]). We do not consider in this paper modular approaches that do not allow for 3D reconfiguration (e.g., Lumen [76], InFORM [26]) or non-selfactuated ones (e.g., GaussBricks [58]). Modules self-actuated in $3 \mathrm{D}$ allow for a larger range of shapes and for system's shape output $[41,86]$. In addition, while non-self-actuated modules can reach small sizes (e.g., 2mm [34]), it is difficult for users to accurately manipulate very small modules. Previous work explored applications enabled by such modules, e.g., computer-assisted design on the physical prototype itself [91], animation directly with a physical prop [64, 83], adapting the interface to the users' needs [82]. This modular approach promises to allow in the future for a large range of shape-change, while enabling high resolution and scalability [2].

The problem we tackle in this paper is to find the range of usable sizes of modules for modular shape-changing physical UIs (PUIs) (i.e. a physical object that the user can manipulate, as opposed to graphical UIs. Previous work stress the importance of this problem [54]. A first reason is that the smallest possible modules are desirable, 
as they allow for instance for the highest possible resolution for the system's shape output modality [54, 77]. A second reason for this importance is that the largest possible modules is desirable to ease their fabrication. In particular, the size of modules was found to have the most impact on other technological design parameters, such as the computational and interaction capabilities embedded in each module [77]. As a consequence, the size of modules suffers from a trade-off between usability and technological feasibility. This paper aims at providing a very first measure of usability to inform future technological development about this trade-off.

A difficulty -and motivation- of this problem lies in the fact that current technology is not yet ready for very small self-actuated modules. Figure 2 shows the modular shape-changing systems that can reconfigure in $3 \mathrm{D}$ and that reached a size smaller than $20 \mathrm{~cm}$ [77]. For instance, the smallest modules studied in HCI are the $9 \mathrm{~mm}$ DynaBlocks [91] (Figure 2). However, Dynablocks are not selfactuated, preventing the system to actuate their 3D display unless they lie on the dedicated table. Major technological advances are done in the autonomous robotics community, where the currently most advanced robotic modules being assembled are the $\varnothing 3.6 \mathrm{~mm}$ Catoms [94] (Figure 2). Figure 2 shows that it is not possible yet to prototype high-resolution modular shape-changing UIs to conduct user studies. It is therefore difficult for the research community to know how far technological research should go to decrease the size of modules in order to provide usable interaction, as we do not know how much the size of the modules impact the interaction.

To solve this difficulty and begin to study the impact of the size of modules on interaction, our approach in this paper is to hypothesize that this technology is close to enable shape-changing PUIs. Based on this hypothesis, we study modular non-shape-changing UIs with non-actuated mock-ups of modules based on the current design of this technology. This allows us to start evaluating the impact of the size of modules between shape-changing phases. As HCI researchers, we believe user-centered design should drive technological development as early as possible [42].

Another difficulty -and grand challenge [2]- lies in the fact that the shape of the modules can also impact user experience with modular UIs $[2,77]$. We chose to start our research with quasi-spherical modules, as this shape (1) is the most promising for miniaturization of actuated robotic modules [74] and (2) is expected to have the most impact on interaction due to the friction caused at the surface of the UI by the gaps between modules.

First, as shown in Figure 2, while the research community mostly explores cubic modules, the robotics community found that quasispherical shape is a promising approach to decrease the size of modules [74]. Indeed, the technologies used to actuate cubic implementations are difficult to miniaturize (e.g., electro-magnets [104], mechanical hinges [64] or flywheels [80]), whereas the rounded edges provided by quasi-spherical modules lower the energy as necessary for miniature actuation [74]. Quasi-spherical shape is therefore a promising research direction for modular shape-changing UIs. For this reason, the impact of this shape should be assessed.

Second, we expect a high difference in user experience between small and large rounded modules, as the size and number of gaps between rounded modules are correlated with the size of the modules. In contrast, we expect a lower difference in user experience between small and large cubic modules, as only the number of gaps is correlated with the size of the modules. While we expect quasispherical modules to hinder performance of common tasks such as target acquisition and pursuit, we do not know to which extent their size will hinder performance. This paper addresses this important problem to re-center technological development on users.

We present the results of two studies assessing four sizes of modules constituting a PUI (namely a slider): a plain, smooth slider (modules of size $\sim 0 \mathrm{~mm}$ ) and three sliders made of $\varnothing 1 \mathrm{~mm}, \varnothing 2.5 \mathrm{~mm}$ and $\varnothing 5 \mathrm{~mm}$ mock-up modules. We find that (1) the largest modules $(\varnothing 5 \mathrm{~mm})$ significantly impairs performance for a pursuit task and subjective perception for pursuit and target acquisition tasks, (2) performance increases when the size of modules decreases but (3) users reportedly enjoyed the haptic feedback of $\varnothing 1 \mathrm{~mm}$ and $\varnothing 2.5 \mathrm{~mm}$ modules (e.g., to brake, change speed and change direction). These results provide deeper understanding on how users interact with a device made of small, rounded modules, to inform future technological goals for shape-changing PUIs.

\section{RELATED WORK}

The study of the size of rounded modules constituting a PUI builds on previous work in PUIs, haptics and psychophysics.

Haptic perception of PUIs. The haptic feedback of an interface involves two kinds of perception: First, the kinesthetic perception is the perception of forces and positions by the muscles and joints [18]. Second, the tactile perception is the perception of the physical properties of an object when in contact with the user's skin [67]. When interacting with a PUI, both kinesthetic and tactile perceptions come into play. For example, users will perceive the shape and roughness of a slider (tactile perception) and the force required to move its cursor (kinesthetic perception).

Previous works distinguish two dimensions of texture: micro (or fine) and macro [67]. Despite differences in perceptual mechanisms, micro- and macro-roughness are hard for users to separate [67]. Prior work studied roughness with groove widths of $1 \mathrm{~mm}$ up to $8 \mathrm{~mm}$ [50], which is in the order of magnitude of the size of the gaps between the modules constituting future reconfigurable PUIs (e.g., $[26,64,91,101])$. On the kinesthetic level, surfaces with groove width of $5 \mathrm{~mm}$ or $8 \mathrm{~mm}$ give a kinesthetic feeling of detents -i.e. the feeling that the cursor is locked at a position, often spring-loaded to make the cursor move toward this position [8]- but are also on the roughness spectrum [50]. Surfaces with smaller groove width, and even micro-levels of roughness, may not give the same feeling of detents but still involve kinesthetic perception through proprioception [102]. Although we expect the size of modules to fall into the macroroughness category (i.e., textures with spatial periods $>200 \mu \mathrm{m}$ ) [53], the surface of the modules may present micro-roughness characteristics (i.e., textures with spatial periods $<200 \mu \mathrm{m}$ ). We conducted a pilot comparing a smooth manufactured slider and the smooth 3D printed one of Figure 1 showing micro-roughness to evaluate how it might impact the results of our experiment. We found no difference. For this reason, this paper focuses on the macro-roughness caused by modules.

Tactile discrimination of textures. The haptics literature recognizes three fundamental dimensions of tactile perception: warmness (e.g., wood vs. metal), hardness (e.g., metal vs. foam) and roughness 


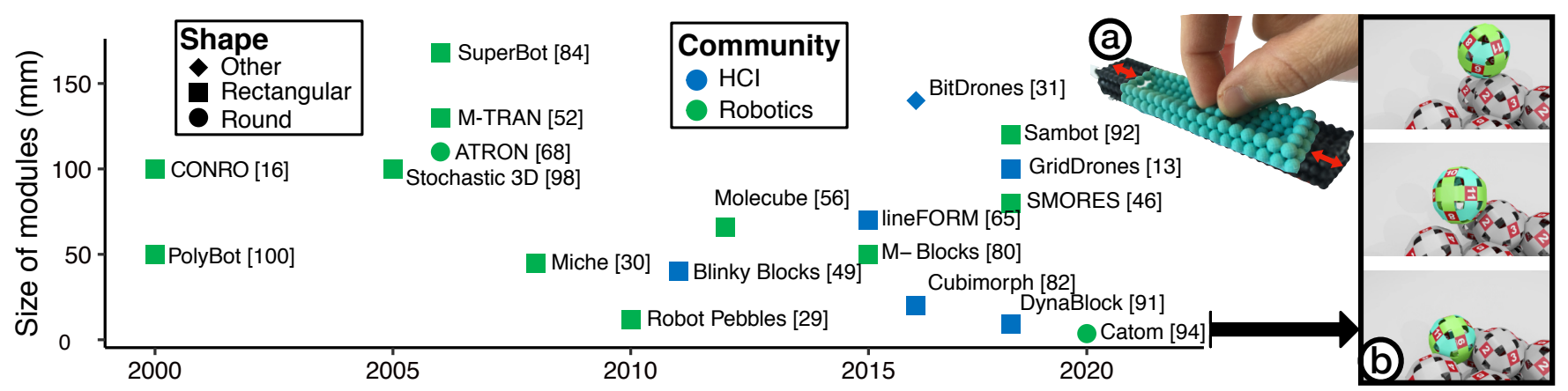

Figure 2: Left: Evolution over time of the size and shape of modules used for modular 3D shape-changing UIs $<20 \mathrm{~mm}$ (data source and references [77] found at http://molecularhci.imag.fr). Right (a): In the future, modular PUIs could be able to reconfigure their shape. For instance here, a slider could become larger [19]. Right (b): The simulated robotic movement of small, interchangeable modules currently developed that could allow such changes in shape [73].

(e.g., sand paper is rough vs. plain paper is smooth) [67]. We particularly review prior work on roughness, as small modules impact the roughness of the interface. When users manipulate PUIs such as a slider, their fingers can (1) stick to the surface of the object, such as when fingers grasp the cursor of the slider. Their fingers can also (2) move tangentially to the surface of the object, such as when fingers swipe a surface. Their fingers can also (3) move a proxy (also called a probe) tangentially to the surface of the object, such as when manipulating an object on a surface or the cursor on its support surface. We thus report results on tactile perception through direct touch with (1) static and (2) moving fingers (e.g., [37, 63, 72, 89]), and through moving probes (3) (e.g., [50, 53, 102, 103]).

The perception of roughness is primarily driven by changes in groove width [50,55]. Human tactile perception of textures with moving fingers heavily depends on the size of the gaps between bumps [14], rather than the size of the bumps. The deeper the skin of the finger can penetrate into the groove between tactile modules, the rougher the user perceives the surface. Specifically, the perception of roughness (1) increases with the distance between the raised tactile modules, (2) increases with the height of the raised modules and, (3) modestly decreases when the size of the raised modules increases [89].

Minimal size of gaps between modules. Users better perceive roughness when they perform tangential movements [61, 89], such as when they perform target acquisition or pursuit with a PUI. The tactile discrimination of two points is more accurate when the fingers move across the surface of the object: the finger's static twopoints discrimination normal ability is $<6 \mathrm{~mm}$ and its moving twopoints discrimination normal ability is $<2 \mathrm{~mm}$ [39, pp.146-148] [22, pp.118-121]. We will not study gaps $>6 \mathrm{~mm}$ because humans are able to discriminate two points $>6 \mathrm{~mm}$ away from each other, even when their finger is static; Also, robots are already research at sizes $<6 \mathrm{~mm}$. Additionally, increasing the surface roughness impacts the kinesthetic perception of friction $[67,89]$. When the gaps and bumps of a rough surface are repeated in a 2D texture, such as with abrasive paper [38], the thresholds are even lower. Through static contact of the finger, users perceive textures if the size of their modules is $>0.1 \mathrm{~mm}$ [38]. With moving contact, human tactile perception is possible for modules of sizes $<0.1 \mathrm{~mm}$ [38], extending to amplitudes as small as $10 \mathrm{~nm}$ for wrinkled surfaces [88]. Based on this we will study modules as small as allowed by standard 3D printers available to the general public. Overall, prior work primarily focus on the perception of roughness, rather than on its impact on another, primary task, such as target acquisition and pursuit, as we do in this paper.

Tactile perception through a probe. The literature on haptics and psychophysics studied the perception of roughness through a probe, i.e. the tip of a stylus (e.g., [50, 53, 102, 103]). E.g., with a tip of size $3 \mathrm{~mm}$, humans are able to perceive gaps of size as small as $0.125 \mathrm{~mm}$. However, this work does not study task performance on a textured surface through a probe, nor through a textured probe or through a probe with a different and larger shape. Manipulating two rough objects on each others, e.g. a rough slider cursor on a rough railing, results in multiple contact points covering a larger contact surface. We do not know yet how these previous results would apply to larger objects and how they relate to common tasks such as target acquisition and pursuit. This is however important to assess to help the design of future modular shape-changing PUIs. Prior work also proposed tools to capture and generate textures through an active haptic probe and tested the correct perception of the captured then regenerated textures [20]. In contrast, our work aims at assessing the impact on task performance of the texture resulting from small modules constituting the PUI.

Tactile perception of roughness and PUIs. Previous work explored the change of tactile perception of the roughness of the UI. For instance, prior work explores vibrotactile feedback for physical dials [10]. Such feedback can easily be perceived even with a static contact of the finger. It is therefore different from the feedback provided by rough surfaces of reconfigurable PUIs made of small modules. Rough devices with prominent textures are an incentive to touch [63] and can be used in the future design of perceived affordances [66] for reconfigurable PUIs. To our knowledge, the literature in PUI and tangible UIs did not explore roughness beyond its subjective perception and abstract qualities [40]. Tactile perception to perform grasp and lift manipulation has been studied [32]: the shape of the object in the region of contact with the skin has a strong effect on grip and lift tasks. However, we do not know yet how the roughness of the surface of the reconfigurable PUI 
-caused by its small modules- affects the performance of common interactive tasks such as target acquisition and pursuit.

Kinesthetic perception and PUIs. Modular implementation of PUIs will cause friction, as the size of modules is correlated with friction [89]. Previous work explored the change of the kinesthetic perception through programmable friction and movement resistance. Programmable friction improves overall movement time and the time to stop the cursor after entering the target with a tactile graphical slider [57]. Variable movement resistance on a physical slider such as with the FireFader [7] improves performance of eye-free interaction [59] or the kinesthetic visualization and manipulation of sound [93]. In prior work, programmable friction and movement resistance were applied in the area of the target. On the contrary, in this paper we are interested in studying the impact of the roughness, and its resulting friction, on the whole surface of the PUI. Previous work also explored the impact of the resolution of pin-based display on a target pursuit task [79]. However, this work did not explore target acquisition tasks. Another limitation is the single, rather large ( $\varnothing 15 \mathrm{~mm})$ size of pins compared to current, smooth and plain PUIs. In addition, the proposed implementation of a slider does not build on top of current widespread sliders. This makes difficult the generalization of this result to widespread sliders made of smaller round modules arranged in 3D such as the ones of Figure 1 and $2 \mathrm{ab}$.

On the one hand, while prior work inform on the human tactile perception according to the size of the gaps between the modules constituting the reconfigurable PUI, it primarily focuses on direct touch. When interaction through probes was studied, these probes are far from widespread PUIs such as dials and sliders, and the tasks performed by participants are far from the tasks performed with PUIs. On the other hand, prior work studied the impact of friction and movement resistance on target acquisition tasks that are widespread in PUIs. However, the friction and movement resistance are applied in the area of the targets only, on the contrary to future reconfigurable PUIs made of small modules. We could not find previous work assessing how the size of modules might impact the performance or the user experience for such tasks.

\section{EXPERIMENTS}

We conduct two user experiments assessing the impact of the size of rounded modules constituting a PUI when using a physical slider One experiment uses a target acquisition task and the other one a pursuit task. For each task, we assess the performance, and the perceived task difficulty and workload.

\subsection{Method}

3.1.1 Apparatus. We chose to study a plain, smooth slider like current sliders as a control condition, and sliders made of modules of three different sizes. First, $\varnothing 1 \mathrm{~mm}$ is the smallest size we achieved with current mainstream 3D printers. Second, we chose to study $\varnothing 5 \mathrm{~mm}$ spheres, as higher sizes importantly impair the global shape of the PUI. Finally, we chose an intermediate size of $\varnothing 2.5 \mathrm{~mm}$ to uniformly study performance in the $\varnothing 0-5 \mathrm{~mm}$ range.

We 3D printed the sliders. The STL models, printers and materials used for the prototypes are provided in the supplementary material for replicability. Our cursor is based on a manufactured, widespread one (Figure 1) [23]. In order to provide a passive haptic feedback comparable to current sliders, the arrangement of the spheres provides a hole for the rail, in which the cursor moves (Figure 1). To allow for a comfortable manipulation of the slider, we provided a support surface around the mock-up (Figure 1). To log the location of the cursor, we mounted the prototypes of modular cursor and casing of modular sliders on a slide potentiometer, as shown in the supplementary video. For accuracy, we chose a $100 \mathrm{~mm}$-long Bourns PTB0143-2010BPB103 [1] over other sizes and manufacturers, as, for instance, PaletteGear $60 \mathrm{~mm}$-long sliders could not be as accurate as $100 \mathrm{~mm}$-long ones [19]. We connected the potentiometers to a computer through a Teensy 3.6 board running the Firmata firmware. The experimental software runs at $60 \mathrm{fps}$. We choose the Teensy 3.6 board for its high performance and its ability to handle 12 bits of resolution when reading the potentiometer. The supplementary video shows how the prototypes work and how participants used the prototypes during the experiment.

3.1.2 Participants. We recruited 24 participants from the local campus. Participants only took part in one of the experiments. Twelve participants performed the target acquisition task (4 female, 8 males, $M=31.33$ y.o., $S D=5.69)$ and the other twelve participants performed the pursuit task ( 4 female, 8 males, $M=30.8$ y.o., $\mathrm{SD}=$ 7.6). All were occasional users of physical sliders.

3.1.3 Experimental design. Target acquisition and pursuit tasks are common in the literature for physical interaction $[25,44,79,95]$. The within-subject independent variable was the size of the modules: Smooth, i.e. no modules (plain 3D printed), spheres of $\varnothing 1 \mathrm{~mm}$, spheres of $\varnothing 2.5 \mathrm{~mm}$, and spheres of $\varnothing 5 \mathrm{~mm}$. The different levels of sizes were presented in permuted order (latin square).

3.1.4 Procedure. The experimenter verifies the tactile sensitivity of the dominant hand's thumb, index and middle fingers through twopoints discrimination test $[22,39]$ and the Semmes-Weinstein monofilament aesthesiometer [6]. There was no sensitivity impairment susceptible to skew the results. The participant then sits in front of a laptop running the experimental software and is presented with the first prototype, on the side of their dominant hand. The participant adjusts the location of the box to comfortably reach the highest and lowest values of the slider. The experimenter secures the location of the box with blu tack. The participant then performs the task. For both tasks, the user controls a $1 \mathrm{~mm}$-thick (4.4px) on-screen cursor with their dominant hand, along an $180 \mathrm{~mm}$-long (800px) vertical axis displayed on-screen (Figure 3). As the potentiometer measures $100 \mathrm{~mm}$, the on-screen cursor moves faster than the physical one with a CD gain of 1.8. The transfer function is a linear mapping.

The participants do not wear earplugs to cancel the noise caused by the friction: We focus in this very first study on the measure of the impact of the size of the modules. As suggested in [69], we leave for future work the identification of the factors causing this impact, such as, e.g., the friction, or the noise caused by this friction.

Target acquisition task. A blue rectangle acting as a target is shown on the vertical axis (Figure 3). The widths (W) and distances (D) of the targets are randomized (W=8px or W=35px, D=90px or $\mathrm{D}=400 \mathrm{px}$ ) and result in three rounded Fitts' Index of Difficulty (ID): 1.8, 3.6 and 5.7. The target becomes green when the cursor is within the target. The participant validates by pressing the space key of a 


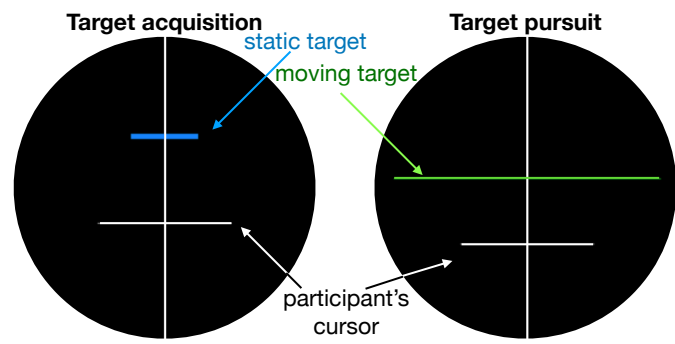

Figure 3: Close-ups of the UI of the experimental software for the target acquisition (left) and pursuit tasks (right).

laptop with their non-dominant hand. If they validate outside the target, we log an error. The task ends when the target is successfully acquired. After completing a training with 5 targets, the participant is required to successfully validate 5 blocks of 26 targets. As the distance (D) is the distance between two consecutive targets, the first and last targets are dummies to re-position the cursor at the center of the screen. Thus, the first and last targets are discarded during analysis. Participants can take breaks between blocks. Instructions require the participant to be as quick and accurate as possible. To account for speed-accuracy trade-off, the experimental software compute the error rate and instruct the participant to speed up (error rate $<4 \%$ ) or slow down $(>4 \%$ ) between blocks.

Target pursuit task. A $1 \mathrm{~mm}$-thick (4.4px) green target is moving up and down the vertical axis (Figure 3). The target moves at constant speed in a linear motion and darts off at pseudo-random intervals (as in [44, 79]). Instructions require the white cursor, controlled by the user, to be as close as possible to the green target. Participants complete two trials of $90 \mathrm{~s}$, as in [25, 44, 79]. They can take a break between trials.

After completion of the task, the participant fills a questionnaire. The procedure is repeated with each of the four sizes of modules. At the end of the experiment, the participant fills a final questionnaire. We conducted interviews after the task using the explicitation interview method [96]. The experiment lasts around 30 minutes.

3.1.5 Data collection and analysis. For the target acquisition task, we collect movement time, error rate and distance from target center to the cursor. For the target pursuit task, we collect the pursuit error for every frame during the experiment. For both, we gather subjective feedback: First, we gather overall perceived difficulty with a Single Ease Question [97] (Overall, how difficult or easy did you find this task?). The participant answers on a seven points Likert scale. Second, we gather perceived workload with a NASA Task Load Index (TLX) questionnaire which assesses six items: mental, physical and temporal demand, performance, effort, and frustration [36]. We use the unweighted version which is as reliable but faster to complete [35, 62]. We also record synchronized videos of the participants' arms, hands and eyes. The experimenter took note of used limbs and user behaviour.

We chose to compare the impact of the size of the modules on our chosen measures as in previous work [21,51]. Other widespread experimental procedures (e.g., two-alternative forced choice [9], free-magnitude estimation [38], or just-noticeable difference [43]) require to many sizes of spheres or only assess the impact on a subjective measure.

\begin{tabular}{|c||ccc|}
\hline & Movement Time $(\mathrm{ms})$ & Error rate (\%) & SEQ \\
\hline \hline Smooth & $1441.29 \pm 623.23$ & $2.28 \pm 14.95$ & $6.01 \pm 1.16$ \\
\hline$\varnothing 1 \mathrm{~mm}$ & $1471.04 \pm 660.18$ & $3.59 \pm 18.61$ & $5.25 \pm 1.36$ \\
\hline$\varnothing 2.5 \mathrm{~mm}$ & $1506.15 \pm 594.66$ & $3.12 \pm 17.39$ & $5.34 \pm 1.15$ \\
\hline$\varnothing 5 \mathrm{~mm}$ & $1508.95 \pm 737.33$ & $1.94 \pm 13.81$ & $4.92 \pm 1.5$ \\
\hline
\end{tabular}

Table 1: Mean movement time $(\mathrm{ms})$, error rate $(\%)$ and score of the Single Ease Question for target acquisition ( \pm stdev).

\subsection{Results - Target acquisition}

In this section, we report our results on the impact of the size of modules constituting the slider on the performance of users performing a target acquisition task. We particularly report in table 1 our results on movement time, error rate and SEQ score.

3.2.1 Performance. While our participants were asked to aim at a $4 \%$ error rate, we wanted to account for any residual bias in speedaccuracy trade-off. A common approach is to use the effective width [90]. However, in order to measure actual movement times, we chose in our experimental procedure to measure the movement time until successful target acquisition as in [27]. In this case, we can directly analyze movement times and error rates separately [17].

Movement Time. Figure 4 shows the geometric mean of the movement time [85] for each size of modules, with its $95 \%$ confidence interval. Since we obtain a p-value of 0.021 with ShapiroWilk's test on our data, we cannot assume the normality of our data. We therefore perform an Aligned Rank Transform (ART) [99]. We conducted a one-way analysis of variance (ANOVA) on the aligned data and showed no significant difference between the movement times of the different sizes of modules $(F(3,33)=1.81, p>.05)$.

Error Rate. Since we obtain a p-value of 0.0003 with ShapiroWilk's test on our data, we cannot assume the normality of our data. We therefore align our data with the ART method. We conducted a one-way analysis of variance (ANOVA) on the aligned data and showed no significant difference between the error rate of the different sizes of modules $(\mathrm{F}(3,33)=1.67, \mathrm{p}>.05)$.

3.2.2 Questionnaires. As questionnaires provide Likert scale data, we used the ART method to align their data, e.g., the mean SEQ scores. We conducted a one-way ANOVA to compare the impact of the size of modules on the aligned SEQ score. As there was significance $(\mathrm{F}(3,33)=4.0637, \mathrm{p}<.05)$, we carried out post-hoc pairwise comparisons based on Kenward-Roger approximation. Participants perceived the task as significantly more difficult with the slider made of $\varnothing 5 \mathrm{~mm}$ modules compared to the smooth slider $(\mathrm{p}<.05)$.

We conducted a one-way ANOVA to compare the impact of the size of modules on each aligned TLX item score and found no significant differences between the TLX items of the different sizes of modules. The raw scores for the SEQ and TLX questionnaires can be found in the supplementary material.

\subsection{Results - Target pursuit}

In this section, we report our results on the impact of the size of modules on the performance of users performing a target pursuit task. We particularly report in table 2 our results on pursuit error and SEQ score. 

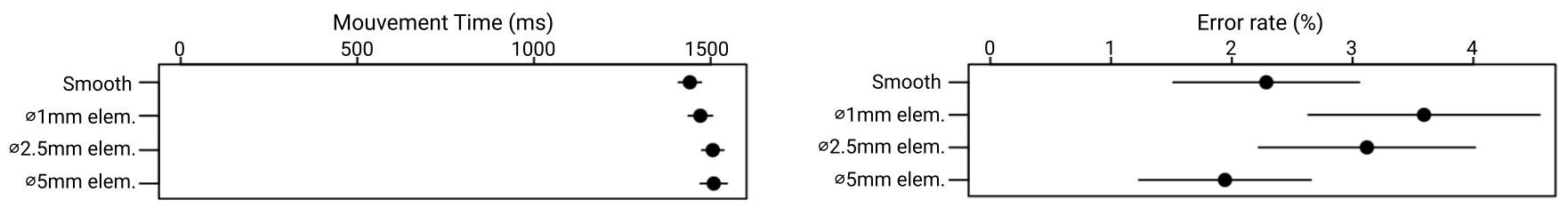

Figure 4: Left: Movement time (in ms) for each size of modules. Points show geometric means and error bars show the $95 \%$ confidence interval. Right: Error rate (\%) for each size of modules. Points show means and error bars show the $95 \%$ confidence interval. All conditions had the same slider range on the device $(8 \mathrm{~cm})$ and on the screen $(18 \mathrm{~cm})$.

\begin{tabular}{|c||ccc|}
\hline & Pursuit error (\%) & Pursuit error (mm) & SEQ \\
\hline \hline Smooth & $1.27 \pm 10.31$ & $2.29 \pm 18.56$ & $5.42 \pm 1.08$ \\
\hline$\varnothing 1 \mathrm{~mm}$ & $1.32 \pm 10.21$ & $2.38 \pm 18.38$ & $4.33 \pm 1.37$ \\
\hline$\varnothing 2.5 \mathrm{~mm}$ & $1.42 \pm 10.06$ & $2.56 \pm 18.11$ & $4.5 \pm 1.24$ \\
\hline$\varnothing 5 \mathrm{~mm}$ & $1.68 \pm 10.39$ & $3.02 \pm 18.70$ & $3.83 \pm 1.80$ \\
\hline
\end{tabular}

Table 2: Median pursuit error (\% and $\mathrm{mm}$ ) and mean score of the Single Ease Question for pursuit ( \pm stdev).

3.3.1 Performance. Figure 5 shows the median pursuit error for each size of modules. The median of the pursuit error is a better indicator of the data central tendency [19]. Since we obtain a pvalue $<0.001$ with Barlett's test on our data, we cannot assume the normality of our data. We aligned our data using ART [99]. We then conducted a one-way analysis of variance (ANOVA) on the aligned data that showed a significance difference $(\mathrm{F}(3,33)=17.879$, $\mathrm{p}<.01)$. We carried out post-hoc pairwise comparisons based on Kenward-Roger approximation [47] and showed two significant increase in pursuit error: First, the slider made of $\varnothing 5 \mathrm{~mm}$ modules lead to significantly more error compared to all other sizes (smooth $(\mathrm{p}<.0001), \varnothing 1 \mathrm{~mm}$ modules $(\mathrm{p}<.0001)$ and $\varnothing 2.5 \mathrm{~mm}$ modules $(\mathrm{p}<.01))$. Second, the slider made of $\varnothing 2.5 \mathrm{~mm}$ modules lead to significantly more error compared to the smooth slider $(\mathrm{p}<.05)$.

3.3.2 Questionnaires. We followed the same statistical approach as for the questionnaire data of the target acquisition task. We compare the impact of the size of modules on the aligned SEQ score. As there was significance $(\mathrm{F}(3,33)=2.978, \mathrm{p}<.05)$, we carried out posthoc pairwise comparisons. The task was perceived as significantly more difficult with the slider made of $\varnothing 5 \mathrm{~mm}$ modules compared to the smooth slider $(\mathrm{p}<.05)$. We compare the impact of the size of modules on each aligned TLX item score. There was a significant impact of the size of modules on the perceived performance $(\mathrm{F}(3,33)=3.9714, \mathrm{p}<.05)$. Post-hoc pairwise comparisons show that the slider made of $\varnothing 5 \mathrm{~mm}$ modules significantly decreased the perceived performance compared to the smooth slider $(\mathrm{p}<.01)$. Results from the final questionnaire comparing the size showed that all users ranked the slider made of $\varnothing 5 \mathrm{~mm}$ modules last for the SEQ as well as for the physical demand ("The task was physically demanding.") and frustration items ("I felt discouraged/irritated while performing the task.") of the TLX. The raw scores for the SEQ and TLX questionnaires are available in the supplementary material.

\section{DISCUSSION}

\subsection{Size of modules \& target acquisition}

Experimental results showed that, even though increasing the size of the modules did not significantly impact performance, the task

was perceived as significantly harder with the $\varnothing 5 \mathrm{~mm}$ modules. This is consistent with participant feedback from interviews. Only two participants expressed liking the slider made of $\varnothing 5 \mathrm{~mm}$ modules (P14 "the large notches helped me to be accurate", P17 "I like the [slider made of $\varnothing 5 \mathrm{~mm}$ modules ] because I think I didn't make any error with it"). The other participants expressed disliking the slider made of $\varnothing 5 \mathrm{~mm}$ modules, as they reportedly perceived it as frustrating: it is too loud (e.g., P21 "the noise is annoying") and is physically uncomfortable to operate (e.g., P20 "it was annoying to move it" and P24 "it's just to the touch, without moving it. I don't like big spheres.").

Overall, participants agreed that all sliders were similar but had distinctive qualities. On the one hand, the smooth slider allows for faster movements and accurate adjustments. However, participants complained that it led them to overshoot more. On the other hand, the sliders made of $\varnothing 1 \mathrm{~mm}$ to $\varnothing 2.5 \mathrm{~mm}$ modules provide haptic feedback which helped to prevent overshoots and accurately stop on the target. Even though participants reportedly enjoyed the sliders made of $\varnothing 1 \mathrm{~mm}$ to $\varnothing 2.5 \mathrm{~mm}$ modules, most still rated the smooth slider as easiest to complete the task with. Our hypothesis is that even though the smooth slider causes overshoots, its increased speed of movement to initially reach the target compensates for the adjustment time required by the overshoot. Participants expressed that, despite their preferences, they did not think any size of modules significantly impacted their performance. For example, P20 said that with the slider made of $\varnothing 5 \mathrm{~mm}$ modules, "completing the task was no problem, I just didn't like it".

Previous work found that programmable friction improves the time to stop the cursor after entering the target and improves overall movement time for non-physical graphical sliders controlled through direct touch [57]. This is consistent with our results with physical sliders: in prior work [57], as friction was only applied on the target, users benefit from (1) the faster movements enabled by low friction to initially reach the target and (2) the higher friction on the target to reduce overshoots. Our results are important as the HCI community takes a growing interest in new shapes, materials and textures [2, 40,75], in particular for reconfigurable PUIs made of small modules (e.g., [26, 31, 54, 58, 64, 91, 101]). Future PUIs can leverage materials that can change roughness or size of modules, such as wrinkling materials (e.g., $[28,71])$ with changing zero-crossing physical feature [81]. It is important that future materials are able to change roughness only in dedicated, programmable locations corresponding to potential targets, e.g. to prevent overshoots while ensuring a comfortable interaction, as shown in our target acquisition experiment. 


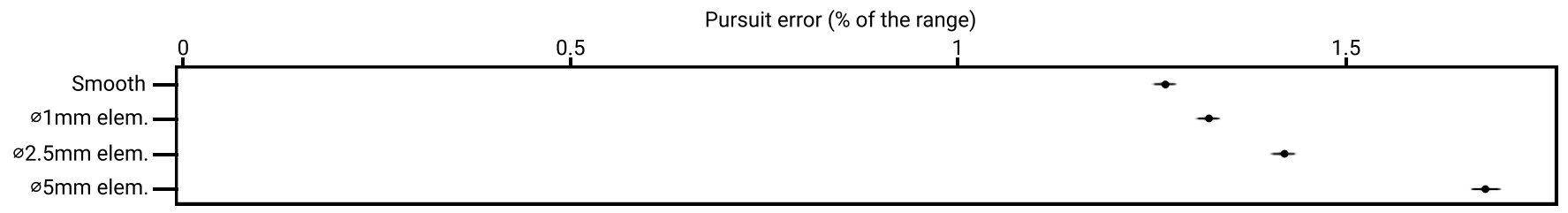

Figure 5: Pursuit error (\% of the slider range). Points show medians and error bars show the $95 \%$ confidence interval. All conditions had the same slider range on the device $(8 \mathrm{~cm})$ and on the screen $(18 \mathrm{~cm})$.

\subsection{Size of modules \& target pursuit}

The size of modules impacts target pursuit more than acquisition. Participants were less accurate at pursuit as the size increased. As the size of modules is correlated with friction [89], we explained this result with the varying friction between the cursor and the rail of the slider. During interviews, participants commented on the relationship between friction, size of modules and performance. On the one hand participants mentioned preferring the plain slider for continuously pursuing the target, whereas it caused overshoots when the target darted off. On the other hand, participants mentioned that larger modules provided haptic feedback. They preferred the sliders made of $\varnothing 1 \mathrm{~mm}$ to $\varnothing 2.5 \mathrm{~mm}$ modules when the target darted off, and said these rendered braking, changing speed and changing direction easier, thus preventing overshoots. This is consistent with the results and user feedback of the target acquisition task.

Even though participants liked the haptic feedback provided by modules, the performance suffered from the large "bumps" made by $\varnothing 5 \mathrm{~mm}$ modules. When the cursor is in a gap between bumps, it is difficult to move because of static friction [78] and because of the deep and steep slope for the cursor to climb the next module. Our results thus support previous work advocating for lower friction [3, $4,12,78]$, probably because the friction resulting from modules is high compared to prior work that found no effect [15].

\subsection{Grasps observed}

Physical sliders allow for several grasps. In particular, when the finger is on the central concave curve of the cursor ("curve" grasp, Figure 6), users can simultaneously control several sliders [5, p.140]. Thus, supporting this "curve" grasp is important. To explore the suitability of a modular implementation for this grasp, we report how participants grasp the cursor in the video recordings of both target acquisition and pursuit tasks. We observed between and within subject variation in grasps. Participants first tried multiple grasps for a same slider. We only report here the grasp they settle on after the first target acquisition block or pursuit trial. We categorize the grasps we found in the three following categories of common grasps for physical sliders [23] (Figure 6, left):

Curve is the grasp of the cursor with a single finger placed in the cursor's central concave curve;

Pinch is the grasp of the cursor between two fingers, often the index and the thumb;

Curve+Pinch is the grasp combining a pinch between one or two finger(s), often the middle and the thumb, and another finger, often the index, in the cursor's concave curve.

The smooth slider and the slider with $\varnothing 1 \mathrm{~mm}$ modules present close performance for all three grasps (Figure 6). The slider with $\varnothing 2.5 \mathrm{~mm}$ modules caused a higher error with the Curve grasp for the pursuit task (yellow in Figure 6). The slider with $\varnothing 5 \mathrm{~mm}$ modules (orange in Figure 6) do not allow for the Curve grasp at all, while the Curve+Pinch grasp caused high error. The Pinch grasp showed the best performance, although its accuracy with $\varnothing 5 \mathrm{~mm}$ modules is still far from the other sizes for the pursuit task.

We hypothesize that this is due to the interaction between the size of the modules and the contact forces applied with the Curve grasp. E.g., P5 said about the smooth slider and the slider with $\varnothing 1 \mathrm{~mm}$ modules, that they are a lot smoother so I have to be aware, I have to change the force that I'm applying. P9 said I think that it's the force too, that I'm not pressing on it the same way so it grips less. With the smooth slider, increasing the contact force between the cursor and the railing does not impair the performance since they are both smooth. With $\varnothing 1 \mathrm{~mm}$ modules, it seems that the bumps and gaps are small enough to accommodate the contact forces of a Curve grasp without impairing the performance. With $\varnothing 2.5 \mathrm{~mm}$ modules, the Curve grasp impairs the performance for the pursuit task. Applying rather tangential forces (either with the Curve+Pinch or Pinch grasps) results in better performance. With $\varnothing 5 \mathrm{~mm}$ modules, applying contact forces renders the movement of the cursor challenging. E.g., P5 performs the Curve grasp: "[on the smooth slider] this is easy with one finger. [switches to $\varnothing 1 \mathrm{~mm}$ modules] easy [switches to $\varnothing 2.5 \mathrm{~mm}$ modules] still easy [switches to $\varnothing 5 \mathrm{~mm}$ modules, the cursor gets stuck] no, not easy". The Curve+Pinch grasp shows little improvement for $\varnothing 5 \mathrm{~mm}$ modules. For this reason, most participants used the Pinch grasp: 6/12 for target acquisition and $10 / 12$ for pursuit. Large size of modules $(\varnothing 5 \mathrm{~mm})$ prevents rich grasps opportunities, and causes lower performance and lower satisfaction.

\subsection{Limitations of the studies}

These studies are limited to the impact of the macro-texture of quasispherical modules. Depending on the future actual implementation, their micro-texture, the strength of their bond, the friction at their surface, their shape, their arrangements, etc. might also have an impact on the results. In particular, we left the micro-texture apart for these studies, as a pilot experiment comparing a standard slider and a 3D printed one did not show any difference in performance. However, it is possible that the micro-texture further impairs the interaction when applied at the surface of small modules. We plan to assess this in a future separate experiment when the first implementations are available. The studies presented here are a first and necessary step towards informing the design of small robotic modules for user interaction with modular PUIs. To our knowledge, these are the first studies assessing the design parameters of small modules constituting PUIs. 


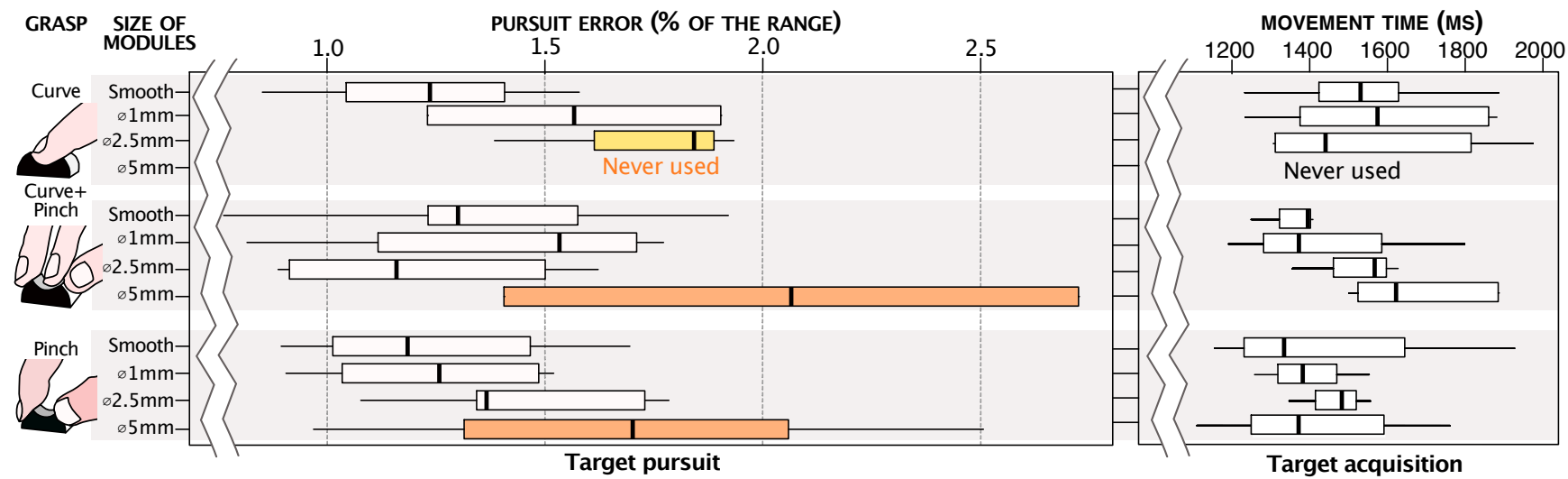

Figure 6: Target pursuit error and target acquisition time for each grasp, across sizes of modules. Boxplots show the median through the bold central line, the first and third quartile through the box and the extremums through the whiskers.

\subsection{Implications}

Modular interfaces will enable the change of the physical shape to support multiple interaction modalities [11] for users' input and/or system's output [2]. We evaluated the impact of the size of rounded modules on target acquisition and pursuit with a slider. Our results already inform the design of rounded modules constituting modular interfaces when they take the shape of a slider.

First, an alternative for future modular reconfigurable PUIs in the future are cubic modules, although such small and actuated cubic modules were never proposed so far. The smallest cubic modules we know to allow for 3D shapes are Dynablocks [91] (9 mm). However these are not self-actuated. We hypothesize that cubic modules would lead to higher target acquisition and pursuit performance compared to rounded ones. Indeed, only the number of gaps is correlated with the size of cubic modules. In contrast, the size and number of gaps between rounded modules are correlated with the size of the rounded modules. Our results can then be used as a worst case scenario for the performance with cubic modules. Future work should assess the precise impact of the size of cubic modules on user experience.

Second, a modular reconfigurable PUI can change to other PUIs besides sliders. Other widespread PUIs are push buttons and dials $[5,48]$. We expect the impact of the size of rounded modules to be comparable for dials, as the rotation of the dial on the support surface will also cause friction. We hypothesize that the contact area between a dial and its support surface (between 78 and $\left.707 \mathrm{~mm}^{2}[5, \mathrm{p} .136]\right)$ is comparable to the contact area between the cursor of the slider and its support surface, as recommended [23] and studied in the paper $\left(231 \mathrm{~mm}^{2}\right)$. Our results also offer a first measure for future modular tangible UIs offering object translation on a table [86]: These objects could change shape in the future thanks to a modular implementation, allowing to change their affordance [60] or the shape of their support surface depending on the scenario of use [33]. Such tangible objects are currently made of large modules [54], but prior work called for decreasing the size of these modules [54], in order to allow for larger shape resolution [81]. Future technological development can now consider user performance to decide for a target size.

\section{CONCLUSION}

We explored the impact of the size of rounded modules for target acquisition and pursuit with future modular PUIs. For target acquisition, our experimental results showed that $\varnothing 1 \mathrm{~mm}$ to $\varnothing 2.5 \mathrm{~mm}$ modules do not significantly impact performance. However, our participants perceived the task as significantly more difficult when using a slider made of $\varnothing 5 \mathrm{~mm}$ modules. Participants found the $\varnothing 5 \mathrm{~mm}$ modules useful to reduce overshoots in eye-free target acquisition. For target pursuit, pursuit accuracy decreases when the size of modules increases, whereas users reportedly prefer sliders with $\varnothing 1 \mathrm{~mm}$ to $\varnothing 2.5 \mathrm{~mm}$ modules to reach darting-off targets. We found that the higher the size of the modules, the fewer the grasps user can perform on the slider's cursor. Impairing the rich grasping possibilities of PUIs can prevent, e.g., their abilities to act as external cognitive aids [45, 87]. Our studies show that the research should consider how the design of these emerging UIs impacts not only user performance and subjective perception, but also the grasping possibilities. Our studies show the importance of addressing the grand challenge [2] of developing $<\varnothing 5 \mathrm{~mm}$ modules.

Future work on reconfigurable PUIs made of modules $<\varnothing 5 \mathrm{~mm}$ also include the study of their arrangements in order to change tactile and kinesthetic feedback only in dedicated, programmable locations corresponding to potential targets. Such a material would entice users to touch the interface on particular locations, and prevent overshoots while ensuring a comfortable interaction.

\section{ACKNOWLEDGMENTS}

This work was supported by the French National Research Agency (ANR-15-CE23-0011) and the LabEx PERSYVAL-Lab (ANR-11-LABX0025-01) funded by the French program Investissement d'avenir. We would like to thank Thomas Achard for providing the data in Figure 2. We are grateful for the feedback we received from colleagues and reviewers.

\section{REFERENCES}

[1] 2019. PTB Series - Low Profile Slide Potentiometer. https://www.bourns.com/ docs/product-datasheets/ptb.pdf?sfvrsn=11289f1 7 .

[2] Jason Alexander, Anne Roudaut, Jürgen Steimle, Kasper Hornbæk, Miguel Bruns Alonso, Sean Follmer, and Timothy Merritt. 2018. Grand Challenges 
in Shape-Changing Interface Research. In the CHI 2018 Conference. New York, New York, USA, 1-14. https://doi.org/10.1145/3173574.3173873

[3] Myroslav Bachynskyi, Gregorio Palmas, Antti Oulasvirta, Jürgen Steimle, and Tino Weinkauf. 2015. Performance and Ergonomics of Touch Surfaces: A Comparative Study Using Biomechanical Simulation. In Proceedings of the 33rd Annual ACM Conference on Human Factors in Computing Systems (Seoul Republic of Korea) (CHI '15). ACM, New York, NY, USA, 1817-1826. https: //doi.org/10.1145/2702123.2702607

[4] Ravin Balakrishnan and I. Scott MacKenzie. 1997. Performance Differences in the Fingers, Wrist, and Forearm in Computer Input Control. In Proceedings of the ACM SIGCHI Conference on Human Factors in Computing Systems (Atlanta, Georgia, USA) (CHI '97). ACM, New York, NY, USA, 303-310. https://doi.org/ $10.1145 / 258549.258764$

[5] Thomas B. Baumann, K. 2001. User Interface Design of Electronic Appliances. Londo: CRC Press. https://doi.org/10.1201/b12625

[6] J. A. Bell. 1978. Sensibility evaluation. Rehabilitation of the hand (1978).

[7] Edgar Berdahl and Alexandros Kontogeorgakopoulos. 2013. The Firefader: Simple, Open-Source, and Reconfigurable Haptic Force Feedback for Musicians. Comput. Music 7. 37, 1 (March 2013), 23-34. https://doi.org/10.1162/COMJ_a_ 00166

[8] Edgar Berdahl, Julius O. Smith, Stefan Weinzierl, and Gunter Niemeyer. 2013. Force-Sensitive Detents Improve User Performance for Linear Selection Tasks. 6, 2 (2013), 206-216. https://doi.org/10.1109/TOH.2012.55

[9] Sanjiv K. Bhatia, Vasudevan Lakshminarayanan, Ashok Samal, and Grant V. Welland. 1995. Human Face Perception in Degraded Images. F. Visual Communication and Image Representation 6 (1995), 280-295.

[10] Andrea Bianchi, Ian Oakley, Jong Keun Lee, Dong Soo Kwon, and Vassilis Kostakos. 2011. Haptics for tangible interaction: a vibro-tactile prototype. (2011) 2.

[11] Jullien Bouchet, Laurence Nigay, and Thierry Ganille. 2004. ICARE Software Components for Rapidly Developing Multimodal Interfaces. In Proceedings of the 6th International Conference on Multimodal Interfaces (State College, PA, USA) (ICMI '04). Association for Computing Machinery, New York, NY, USA 251-258. https://doi.org/10.1145/1027933.1027975

[12] James V Bradley. 1969. Optimum Knob Diameter. Human Factors: The fournal of the Human Factors and Ergonomics Society 11, 4 (Aug. 1969), 353-360. https: //doi.org/10.1177/001872086901100406

[13] Sean Braley, Calvin Rubens, Timothy Merritt, and Roel Vertegaal. 2018. GridDrones: A Self-Levitating Physical Voxel Lattice for Interactive 3D Surface Deformations. In Proceedings of the 31st Annual ACM Symposium on User Interface Software and Technology (UIST '18). Association for Computing Machinery, New York, NY, USA, 87-98. https://doi.org/10.1145/3242587.3242658

[14] C J Cascio and K Sathian. 2001. Temporal cues contribute to tactile perception of roughness. The fournal of neuroscience : the official journal of the Society for Neuroscience 21, 14 (July 2001), 5289-5296. http://eutils.ncbi.nlm.nih.gov/entrez/ eutils/elink.fcgi?dbfrom=pubmed\&id=11438604\&retmode=ref\&cmd=prlinks

[15] Géry Casiez, Nicolas Roussel, Romuald Vanbelleghem, and Frédéric Giraud. 2011. Surfpad: Riding Towards Targets on a Squeeze Film Effect. In Proceedings of the SIGCHI Conference on Human Factors in Computing Systems (Vancouver, BC, Canada) (CHI '11). ACM, New York, NY, USA, 2491-2500. https://doi.org/ 10.1145/1978942.1979307

[16] Andres Castano, Wei-Min Shen, and Peter Will. 2000. CONRO: Towards Deployable Robots with Inter-Robots Metamorphic Capabilities. Autonomous Robots 8 , 3 (June 2000), 309-324. https://doi.org/10.1023/A:1008985810481

[17] Olivier Chapuis and Pierre Dragicevic. 2011. Effects of motor scale, visual scale, and quantization on small target acquisition difficulty. 18, 3 (2011), 1-32. https://doi.org/10.1145/1993060.1993063

[18] Andy Cockburn and Stephen Brewster. 2005. Multimodal feedback for the acquisition of small targets. 48, 9 (2005), 1129-1150. https://doi.org/10.1080/ 00140130500197260

[19] Céline Coutrix and Cédric Masclet. 2015. Shape-Change for Zoomable TUIs Opportunities and Limits of a Resizable Slider. In Human-Computer Interaction - INTERACT 2015. Springer International Publishing, Cham, 349-366. https: //doi.org/10.1007/978-3-319-22701-6_27

[20] H. Culbertson, J. Unwin, and K. J. Kuchenbecker. 2014. Modeling and Rendering Realistic Textures from Unconstrained Tool-Surface Interactions. 7, 3 (2014), 381-393. https://doi.org/10.1109/TOH.2014.2316797 Conference Name: IEEE Transactions on Haptics.

[21] Andrea De Cesarei and Maurizio Codispoti. 2010. Effects of Picture Size Reduction and Blurring on Emotional Engagement. PLOS ONE 5, 10 (10 2010), 1-7. https://doi.org/10.1371/journal.pone.0013399

[22] A. Lee Dellon. 1997. Somato Sensory Testing and Rehabilitation.

[23] European Committee for Standardization. 2008. Safety of machinery - Ergonomics requirements for the design of displays and control actuators - Part 3: Control actuators.

[24] Brian Feeny, Ardeshir Guran, Nikolaus Hinrichs, and Karl Popp. 1998. A Historical Review on Dry Friction and Stick-Slip Phenomena. Applied Mechanics Reviews 51, 5 (05 1998), 321-341. https://doi.org/10.1115/1.3099008
arXiv:https://asmedigitalcollection.asme.org/appliedmechanicsreviews/articlepdf/51/5/321/4748671/321_1.pdf

[25] George W Fitzmaurice and William Buxton. 1997. An empirical evaluation of graspable user interfaces. In CHI. ACM Press, New York, New York, USA, 43-50. https://doi.org/10.1145/258549.258578

[26] Sean Follmer, Daniel Leithinger, Alex Olwal, Akimitsu Hogge, and Hiroshi Ishii. 2013. inFORM: dynamic physical affordances and constraints through shape and object actuation. In Proceedings of the 26th annual ACM symposium on User interface software and technology - UIST '13 (St. Andrews, Scotland, United Kingdom, 2013). ACM Press, 417-426. https://doi.org/10.1145/2501988.2502032

[27] Clifton Forlines, Daniel Wigdor, Chia Shen, and Ravin Balakrishnan. 2007. Direct-touch vs. mouse input for tabletop displays. In Proceedings of the SIGCHI Conference on Human Factors in Computing Systems - CHI '07 (San Jose, California, USA, 2007). ACM Press, 647-656. https://doi.org/10.1145/1240624.1240726

[28] Jack Forman, Taylor Tabb, Youngwook Do, Meng-Han Yeh, Adrian Galvin, and Lining Yao. 2019. ModiFiber: Two-Way Morphing Soft Thread Actuators for Tangible Interaction. In Proceedings of the 2019 CHI Conference on Human Factors in Computing Systems (Glasgow, Scotland Uk) (CHI '19). Association for Computing Machinery, New York, NY, USA, 1-11. https://doi.org/10.1145/ 3290605.3300890

[29] Kyle Gilpin, Ara Knaian, and Daniela Rus. 2010. Robot pebbles: One centimeter modules for programmable matter through self-disassembly. In 2010 IEEE International Conference on Robotics and Automation. IEEE, Anchorage, AK, 2485-2492. https://doi.org/10.1109/ROBOT.2010.5509817

[30] Kyle Gilpin, Keith Kotay, Daniela Rus, and Iuliu Vasilescu. 2008. Miche: Modular Shape Formation by Self-Disassembly. The International fournal of Robotics Research 27, 3-4 (March 2008), 345-372. https://doi.org/10.1177/0278364907085557

[31] Antonio Gomes, Calvin Rubens, Sean Braley, and Roel Vertegaal. 2016. BitDrones: Towards Using 3D Nanocopter Displays As Interactive Self-Levitating Programmable Matter. In Proceedings of the 2016 CHI Conference on Human Factors in Computing Systems (San Jose, California, USA) (CHI '16). ACM, New York, NY, USA, 770-780. https://doi.org/10.1145/2858036.2858519

[32] Antony W Goodwin and Heather E Wheat. 2004. Sensory signals in neural populations underlying tactile perception and manipulation. Annual review of neuroscience 27 (2004), 53-77. https://doi.org/10.1146/annurev.neuro.26.041002. 131032

[33] Jens Emil Grønbæk, Majken Kirkegaard Rasmussen, Kim Halskov, and Marianne Graves Petersen. 2020. KirigamiTable: Designing for Proxemic Transitions with a Shape-Changing Tabletop. In Proceedings of the $2020 \mathrm{CHI}$ Conference on Human Factors in Computing Systems (Honolulu, HI, USA) (CHI '20). Association for Computing Machinery, New York, NY, USA, 1-15. https: //doi.org/10.1145/3313831.3376834

[34] Hongri Gu, Quentin Boehler, Daniel Ahmed, and Bradley J. Nelson. 2019. Magnetic quadrupole assemblies with arbitrary shapes and magnetizations. Science Robotics 4, 35 (Oct. 2019), eaax8977. https://doi.org/10.1126/scirobotics.aax8977

[35] Sandra G Hart and Moffett Field. 2006. NASA-TASK LOAD INDEX (NASA-TLX); 20 YEARS LATER. (2006), 5.

[36] Sandra G. Hart and Lowell E. Staveland. 1988. Development of NASA-TLX (Task Load Index): Results of Empirical and Theoretical Research. In Advances in Psychology. Vol. 52. Elsevier, 139-183. https://doi.org/10.1016/S0166-4115(08) 62386-9

[37] Mark Holliins, Richard Faldowski, Suman Rao, and Forrest Young. 1993. Perceptual dimensions of tactile surface texture: A multidimensional scaling analysis. 54, 6 (1993), 697-705. https://doi.org/10.3758/BF03211795

[38] M Hollins and S R Risner. 2000. Evidence for the duplex theory of tactile texture perception. Perception \& psychophysics 62, 4 (May 2000), 695705. http://eutils.ncbi.nlm.nih.gov/entrez/eutils/elink.fcgi?dbfrom=pubmed\& id $=10883578 \&$ retmode $=$ ref \& $\mathrm{cmd}=$ prlinks

[39] James M. D. Hunter, Evelyn P. T. Mackin, and Anne M. S. Callahan. 1995. Rehabilitation of the Hand: Surgery and Therapy (4 ed.). Vol. 1.

[40] Jörn Hurtienne and Daniel Reinhardt. 2017. Texture Metaphors and Tangible Interaction: No Smooth Relationship?. In Proceedings of the Tenth International Conference on Tangible, Embedded, and Embodied Interaction - TEI '17 (Yokohama, Japan, 2017). ACM Press, 79-87. https://doi.org/10.1145/3024969.3024986

[41] Hiroshi Ishii, Dávid Lakatos, Leonardo Bonanni, and Jean-Baptiste Labrune. 2012. Radical atoms: beyond tangible bits, toward transformable materials. Interactions 19, 1 (Jan. 2012), 38-51. https://doi.org/10.1145/2065327.2065337

[42] ISO9241-210:2019 2019. Ergonomics of Human-System Interaction - Part 210: Human-Centered Design for Interactive Systems. Standard. International Organization for Standardization, Geneva, $\mathrm{CH}$.

[43] Sungjune Jang, Lawrence H. Kim, Kesler Tanner, Hiroshi Ishii, and Sean Follmer. 2016. Haptic Edge Display for Mobile Tactile Interaction. In Proceedings of the 2016 CHI Conference on Human Factors in Computing Systems (San Jose, California, USA) (CHI '16). ACM, New York, NY, USA, 3706-3716. https://doi. org $/ 10.1145 / 2858036.2858264$

[44] Yvonne Jansen, Pierre Dragicevic, and Jean-Daniel Fekete. 2012. Tangible remote controllers for wall-size displays. In CHI '12: Proceedings of the SIGCHI Conference 
on Human Factors in Computing Systems. ACM, New York, New York, USA, 28652874. https://doi.org/10.1145/2207676.2208691

[45] Yvonne Jansen, Pierre Dragicevic, and Jean-Daniel Fekete. 2013. Evaluating the Efficiency of Physical Visualizations. In Proceedings of the SIGCHI Conference on Human Factors in Computing Systems (Paris, France) (CHI '13). Association for Computing Machinery, New York, NY, USA, 2593-2602. https://doi.org/10. $1145 / 2470654.2481359$

[46] Gangyuan Jing, Tarik Tosun, Mark Yim, and Hadas Kress-Gazit. 2018. Accomplishing high-level tasks with modular robots. Autonomous Robots 42, 7 (Oct. 2018), 1337-1354. https://doi.org/10.1007/s10514-018-9738-1

[47] Michael Kenward and James Roger. 2009. An improved approximation to the precision of fixed effects from restricted maximum likelihood. Computational Statistics \& Data Analysis 53 (05 2009), 2583-2595. https://doi.org/10.1016/j. csda.2008.12.013

[48] Hyunyoung Kim, Céline Coutrix, and Anne Roudaut. 2018. KnobSlider: Design of a Shape-Changing UI for Parameter Control. In CHI '18. https://dl.acm.org/ citation.cfm?id=3173913

[49] Brian T. Kirby, Michael Ashley-Rollman, and Seth Copen Goldstein. 2011. Blinky Blocks: A Physical Ensemble Programming Platform. In CHI '11 Extended Abstracts on Human Factors in Computing Systems (Vancouver, BC, Canada) (CHI EA '11). ACM, New York, NY, USA, 1111-1116. https://doi.org/10.1145/1979742. 1979712

[50] Roberta L. Klatzky, Susan J. Lederman, Cheryl Hamilton, Molly Grindley, and Robert H. Swendsen. 2003. Feeling textures through a probe: Effects of probe and surface geometry and exploratory factors. 65, 4 (2003), 613-631. https: //doi.org/10.3758/BF03194587

[51] Hendrik Knoche, John D. McCarthy, and M. Angela Sasse. 2008. How low can you go? The effect of low resolutions on shot types in mobile TV. Multimedia Tools and Applications 36, 1 (01 Jan 2008), 145-166. https://doi.org/10.1007/s11042006-0076-5

[52] Haruhisa Kurokawa, Kohji Tomita, Akiya Kamimura, Satoshi Murata, Yuzuru Terada, and Shigeru Kokaji. 2006. Distributed Metamorphosis Control of a Modular Robotic System M-TRAN. In Distributed Autonomous Robotic Systems 7, Maria Gini and Richard Voyles (Eds.). Springer Japan, Tokyo, 115-124. https: //doi.org/10.1007/4-431-35881-1_12

[53] Michael A Lawrence, Ryo Kitada, Roberta L Klatzky, and Susan J Lederman 2007. Haptic Roughness Perception of Linear Gratings via Bare Finger or Rigid Probe. 36, 4 (2007), 547-557. https://doi.org/10.1068/p5746

[54] Mathieu Le Goc, Lawrence H Kim, Ali Parsaei, Jean-Daniel Fekete, Pierre Dragicevic, and Sean Follmer. 2016. Zooids: Building Blocks for Swarm User Interfaces. In UIST'16. 1-13. https://doi.org/10.1145/2984511.2984547

[55] S. J. Lederman and R. L. Klatzky. 2009. Haptic perception: A tutorial. 71, 7 (2009), 1439-1459. https://doi.org/10.3758/APP.71.7.1439

[56] Jong-Hyun Lee and Chang Wook Ahn. 2012. Evolutionary self-assembling swarm robots using genetic programming. In 2012 Proceedings of SICE Annual Conference (SICE). 807-811.

[57] Vincent Levesque, Louise Oram, Karon MacLean, Andy Cockburn, Nicholas D. Marchuk, Dan Johnson, J. Edward Colgate, and Michael A. Peshkin. 2011. Enhancing physicality in touch interaction with programmable friction. In Proceedings of the 2011 annual conference on Human factors in computing systems - CHI '11 (Vancouver, BC, Canada, 2011). ACM Press, 2481. https: //doi.org/10.1145/1978942.1979306

[58] Rong-Hao Liang, Liwei Chan, Hung-Yu Tseng, Han-Chih Kuo, Da-Yuan Huang, De-Nian Yang, and Bing-Yu Chen. 2014. GaussBricks: Magnetic Building Blocks for Constructive Tangible Interactions on Portable Displays. In Proceedings of the SIGCHI Conference on Human Factors in Computing Systems (Toronto, Ontario, Canada) (CHI '14). Association for Computing Machinery, New York, NY, USA 3153-3162. https://doi.org/10.1145/2556288.2557105

[59] Lars Lischke, Paweł W. Woźniak, Sven Mayer, Andreas Preikschat, and Morten Fjeld. 2017. Using Variable Movement Resistance Sliders for Remote Discrete Input. In Proceedings of the Interactive Surfaces and Spaces on ZZZ - ISS '17 (Brighton, United Kingdom, 2017). ACM Press, 116-125. https://doi.org/10. $1145 / 3132272.3134135$

[60] Rafael Morales González, Caroline Appert, Gilles Bailly, and Emmanuel Pietriga. 2016. TouchTokens: Guiding Touch Patterns with Passive Tokens. In Proceedings of the 2016 CHI Conference on Human Factors in Computing Systems (San Jose, California, USA) (CHI '16). Association for Computing Machinery, New York, NY, USA, 4189-4202. https://doi.org/10.1145/2858036.2858041

[61] J.W. Morley, A.W. Goodwin, and I. Darian-Smith. 1983. Tactile discrimination of gratings. 49, 2 (1983). https://doi.org/10.1007/BF00238588

[62] W. F. Moroney, D. W. Biers, F. T. Eggemeier, and J. A. Mitchell. 1992. A comparison of two scoring procedures with the NASA task load index in a simulated flight task. In Proceedings of the IEEE 1992 National Aerospace and Electronics Conference. 734-740 vol.2. https://doi.org/10.1109/NAECON.1992.220513

[63] Hikaru Nagano, Shogo Okamoto, and Yoji Yamada. 2014. Haptic Invitation of Textures: Perceptually Prominent Properties of Materials Determine Human Touch Motions. 7, 3 (2014), 345-355. https://doi.org/10.1109/TOH.2014.2321575
[64] Ken Nakagaki, Artem Dementyev, Sean Follmer, Joseph A. Paradiso, and Hiroshi Ishii. 2016. ChainFORM: A Linear Integrated Modular Hardware System for Shape Changing Interfaces. In Proceedings of the 29th Annual Symposium on User Interface Software and Technology (Tokyo, Japan, 2016-10-16) (UIST '16). Association for Computing Machinery, 87-96. https://doi.org/10.1145/2984511. 2984587

[65] K Nakagaki, S Follmer, and H Ishii. 2015. LineFORM: Actuated Curve Interfaces for Display, Interaction, and Constraint. In Proceedings of the 28th Annual ACM Conference on Human Factors in Computing. https://doi.org/10.1145/2807442. 2807452

[66] Donald A. Norman. 1999. Affordance, Conventions, and Design. Interactions 6, 3 (May 1999), 38-43. https://doi.org/10.1145/301153.301168

[67] Shogo Okamoto, Hikaru Nagano, and Yoji Yamada. 2013. Psychophysical Dimensions of Tactile Perception of Textures. 6, 1 (2013), 81-93. https: //doi.org/10.1109/TOH.2012.32

[68] Esben Hallundbæk Østergaard, Kristian Kassow, Richard Beck, and Henrik Hautop Lund. 2006. Design of the ATRON lattice-based self-reconfigurable robot. Autonomous Robots 21, 2 (01 Sep 2006), 165-183. https://doi.org/10.1007/s10514006-8546-1

[69] Antti Oulasvirta and Kasper Hornbæk. 2016. HCI Research as Problem-Solving. In Proceedings of the 2016 CHI Conference on Human Factors in Computing Systems (San Jose, California, USA) (CHI '16). Association for Computing Machinery, New York, NY, USA, 4956-4967. https://doi.org/10.1145/2858036.2858283

[70] Irene Parada, Vera Sacristán, and Rodrigo I. Silveira. 2016. A new metamodule for efficient reconfiguration of hinged-units modular robots. In 2016 IEEE International Conference on Robotics and Automation (ICRA). 5197-5202. https://doi.org/10.1109/ICRA.2016.7487726

[71] Sylvain Pauchet, Jean-Luc Vinot, Catherine Letondal, Alexandre Lemort, Claire Lavenir, Timothée Lecomte, Stéphanie Rey, Valentin Becquet, and Guillaume Crouzet. 2019. Multi-Plié: A Linear Foldable and Flattenable Interactive Display to Support Efficiency, Safety and Collaboration. In Proceedings of the 2019 CHI Conference on Human Factors in Computing Systems (Glasgow, Scotland Uk) (CHI '19). Association for Computing Machinery, New York, NY, USA, 1-13. https://doi.org/10.1145/3290605.3300384

[72] Delphine Picard, Catherine Dacremont, Dominique Valentin, and Agnès Giboreau. 2003. Perceptual dimensions of tactile textures. 114, 2 (2003), 165-184. https://doi.org/10.1016/j.actpsy.2003.08.001

[73] Benoit Piranda and Julien Bourgeois. 2018. Designing a quasi-spherical module for a huge modular robot to create programmable matter. Autonomous Robot fournal, Special Issue: Distributed Robotics: From Fundamentals to Applications 42, 8 (2018), 1619-1633. https://doi.org/10.1007/s10514-018-9710-0

[74] Benoît Piranda and Julien Bourgeois. 2018. Geometrical Study of a Quasispherical Module for Building Programmable Matter. In Distributed Autonomous Robotic Systems, Roderich Groß, Andreas Kolling, Spring Berman, Emilio Frazzoli, Alcherio Martinoli, Fumitoshi Matsuno, and Melvin Gauci (Eds.). Vol. 6. Springer International Publishing, 387-400. https://doi.org/10.1007/978-3-31973008-0 27 Series Title: Springer Proceedings in Advanced Robotics.

[75] Ingrid Maria Pohl and Lian Loke. 2013. Touch toolkit: a method to convey touch-based design knowledge and skills. In Proceedings of the 8th International Conference on Tangible, Embedded and Embodied Interaction - TEI '14 (Munich, Germany, 2013). ACM Press, 251-258. https://doi.org/10.1145/2540930.2540957

[76] Ivan Poupyrev, Tatsushi Nashida, Shigeaki Maruyama, Jun Rekimoto, and Yasufumi Yamaji. 2004. Lumen: interactive visual and shape display for calm computing. In ACM SIGGRAPH 2004 Emerging technologies on - SIGGRAPH '04. ACM Press, Los Angeles, California, 17. https://doi.org/10.1145/1186155.1186173

[77] Laura Pruszko, Céline Coutrix, Yann Laurillau, Benoît Piranda, and Julien Bourgeois. 2021. Molecular HCI: Structuring the Cross-disciplinary Space of Modular Shape-changing User Interfaces. Proceedings of the ACM on Human-Computer Interaction (2021). https://doi.org/10.1145/3461733

[78] Christopher Richard and Mark Cutkosky. 2000. The Effects of Real and Computer Generated Friction on Human Performance in a Targeting Task. In Proceedings of the ASME IMECE Symposium on Haptic Interfaces for Virtual Environments and Teleoperator Systems. ASME, 1101-1108.

[79] Simon Robinson, Céline Coutrix, Jennifer Pearson, Juan Rosso, Matheus Fernandes Torquato, Laurence Nigay, and Matt Jones. 2016. Emergeables: Deformable Displays for Continuous Eyes-Free Mobile Interaction. In CHI '16: Proceedings of the 2016 CHI Conference on Human Factors in Computing Systems. Swansea University, ACM, New York, New York, USA, 3793-3805. https: //doi.org/10.1145/2858036.2858097

[80] John W. Romanishin, Kyle Gilpin, Sebastian Claici, and Daniela Rus. 2015. 3D M-Blocks: Self-reconfiguring robots capable of locomotion via pivoting in three dimensions. In 2015 IEEE International Conference on Robotics and Automation (ICRA). IEEE, Seattle, WA, USA, 1925-1932. https://doi.org/10.1109/ICRA.2015. 7139450

[81] Anne Roudaut, Abhijit Karnik, Markus Löchtefeld, and Sriram Subramanian. 2013. Morphees: toward high "shape resolution" in self-actuated flexible mobile devices. In Proceedings of the SIGCHI Conference on Human Factors in Computing Systems - CHI '13 (Paris, France, 2013). ACM Press, 593. https://doi.org/10.1145/ 
2470654.2470738

[82] A Roudaut, D Krusteva, M McCoy, A Karnik, K Ramani, and S Subramanian. 2016. Cubimorph: Designing modular interactive devices. In 2016 IEEE International Conference on Robotics and Automation (ICRA. IEEE, 3339-3345. https://doi. org/10.1109/ICRA.2016.7487508

[83] Calvin Rubens, Sean Braley, Julie Torpegaard, Nicklas Lind, Roel Vertegaal, and Timothy Merritt. 2020. Flying LEGO Bricks: Observations of Children Constructing and Playing with Programmable Matter. In Proceedings of the Fourteenth International Conference on Tangible, Embedded, and Embodied Interaction (TEI '20). Association for Computing Machinery, New York, NY, USA, 193-205. https://doi.org/10.1145/3374920.3374948

[84] Behnam Salemi, Mark Moll, and Wei-min Shen. 2006. SUPERBOT: A Deployable, Multi-Functional, and Modular Self-Reconfigurable Robotic System. In 2006 IEEE/RSF International Conference on Intelligent Robots and Systems. IEEE, Beijing, China, 3636-3641. https://doi.org/10.1109/IROS.2006.281719

[85] Jeff Sauro and James R. Lewis. 2010. Average Task Times in Usability Tests What to Report?. In Proceedings of the SIGCHI Conference on Human Factors in Computing Systems (Atlanta, Georgia, USA) (CHI'10). ACM, New York, NY, USA, 2347-2350. https://doi.org/10.1145/1753326.1753679

[86] Orit Shaer and Eva Hornecker. 2010. Tangible User Interfaces: Past, Present and Future Directions. Vol. 3. Now Publishers Inc. https://doi.org/10.1561/1100000026

[87] Orit Shaer, Nancy Leland, Eduardo H Calvillo Gámez, and Robert J K Jacob. 2004 The TAC paradigm: specifying tangible user interfaces. Personal and Ubiquitous Computing 8, 5 (2004), 359-369. https://doi.org/10.1007/s00779-004-0298-3

[88] Lisa Skedung, Martin Arvidsson, Jun Young Chung, Christopher M. Stafford Birgitta Berglund, and Mark W. Rutland. 2013. Feeling Small: Exploring the Tactile Perception Limits. 3, 1 (2013), 2617. https://doi.org/10.1038/srep02617

[89] Allan M. Smith, C. Elaine Chapman, Mélanie Deslandes, Jean-Sébastien Langlais, and Marie-Pierre Thibodeau. 2002. Role of friction and tangential force variation in the subjective scaling of tactile roughness. 144, 2 (2002), 211-223. https: //doi.org/10.1007/s00221-002-1015-y

[90] R. William Soukoreff and I. Scott MacKenzie. 2004. Towards a standard for pointing device evaluation, perspectives on 27 years of Fitts' law research in HCI. 61, 6 (2004), 751-789. https://doi.org/10.1016/j.ijhcs.2004.09.001

[91] Ryo Suzuki, Junichi Yamaoka, Daniel Leithinger, Tom Yeh, Mark D. Gross, Yoshihiro Kawahara, and Yasuaki Kakehi. 2008. Dynablock: Dynamic 3D Printing for Instant and Reconstructable Shape Formation. In The 31st Annual ACM Symposium on User Interface Software and Technology - UIST '18 (Berlin, Germany, 2018). ACM Press, 99-111. https://doi.org/10.1145/3242587.3242659

[92] Wenshuai Tan, Hongxing Wei, and Bo Yang. 2018. SambotII: A New SelfAssembly Modular Robot Platform Based on Sambot. Applied Sciences 8, 10 (2018). https://doi.org/10.3390/app8101719

[93] Atau Tanaka and Adam Parkinson. 2016. Haptic Wave: A Cross-Modal Interface for Visually Impaired Audio Producers. Association for Computing Machinery, New York, NY, USA, 2150-2161. https://doi.org/10.1145/2858036.2858304

[94] Pierre Thalamy, Benoit Piranda, and Julien Bourgeois. 2020. 3D Coating SelfAssembly for Modular Robotic Scaffolds. In 2020 IEEE/RS7 International Conference on Intelligent Robots and Systems (IROS). IEEE, Las Vegas, NV, USA, 11688-11695. https://doi.org/10.1109/IROS45743.2020.9341324

[95] Philip Tuddenham, David Kirk, and Shahram Izadi. 2010. Graspables Revisited: Multi-touch vs. Tangible Input for Tabletop Displays in Acquisition and $\mathrm{Ma}-$ nipulation Tasks. In Proceedings of the SIGCHI Conference on Human Factors in Computing Systems (Atlanta, Georgia, USA) (CHI '10). ACM, New York, NY, USA, 2223-2232. https://doi.org/10.1145/1753326.1753662

[96] Pierre Vermersch. 2018. The explicitation interview.

[97] Werner Wetzlinger, Andreas Auinger, and Michael Doerflinger. 2014. Comparing Effectiveness, Efficiency, Ease of Use, Usability and User Experience When Using Tablets and Laptops. Lecture Notes in Computer Science 8517, 402-412. https://doi.org/10.1007/978-3-319-07668-3_39

[98] Paul White, Victor Zykov, Josh Bongard, and Hod Lipson. 2005. Three Dimensional Stochastic Reconfiguration of Modular Robots. In Robotics: Science and Systems I. Robotics: Science and Systems Foundation. https://doi.org/10.15607/ RSS.2005.I.022

[99] Jacob O. Wobbrock, Leah Findlater, Darren Gergle, and James J. Higgins. 2011. The Aligned Rank Transform for Nonparametric Factorial Analyses Using Only Anova Procedures. In Proceedings of the SIGCHI Conference on Human Factors in Computing Systems (Vancouver, BC, Canada) (CHI '11). ACM, New York, NY, USA, 143-146. https://doi.org/10.1145/1978942.1978963

[100] M. Yim, D.G. Duff, and K.D. Roufas. 2000. PolyBot: a modular reconfigurable robot. In Proceedings 2000 ICRA. Millennium Conference. IEEE International Conference on Robotics and Automation. Symposia Proceedings (Cat. No.00CH37065) Vol. 1. IEEE, San Francisco, CA, USA, 514-520. https://doi.org/10.1109/ROBOT. 2000.844106

[101] Shigeo Yoshida, Yuqian Sun, and Hideaki Kuzuoka. 2020. PoCoPo: Handheld Pin-based Shape Display for Haptic Rendering in Virtual Reality. (2020), 13.

[102] Takashi Yoshioka, James C Craig, Graham C Beck, and Steven S Hsiao. 2011. Perceptual Constancy of Texture Roughness in the Tactile System. (2011), 9.
[103] Takashi Yoshioka and Julia Zhou. 2009. Factors Involved in Tactile Texture Perception Through Probes. (2009), 22.

[104] Victor Zykov, Efstathios Mytilinaios, Mark Desnoyer, and Hod Lipson. 2007. Evolved and Designed Self-Reproducing Modular Robotics. IEEE Transactions on Robotics 23, 2 (April 2007), 308-319. https://doi.org/10.1109/TRO.2007.894685 